PROCEEDINGS OF THE

AMERICAN MATHEMATICAL SOCIETY

Volume 134, Number 3, Pages 673-681

S 0002-9939(05)08361-9

Article electronically published on September 28, 2005

\title{
THE SET OF COMMON FIXED POINTS OF A ONE-PARAMETER CONTINUOUS SEMIGROUP OF MAPPINGS IS $F(T(1)) \cap F(T(\sqrt{2}))$
}

\author{
TOMONARI SUZUKI
}

(Communicated by Jonathan M. Borwein)

\begin{abstract}
In this paper we prove the following theorem: Let $\{T(t): t \geq 0\}$ be a one-parameter continuous semigroup of mappings on a subset $C$ of a Banach space $E$. The set of all fixed points of $T(t)$ is denoted by $F(T(t))$ for each $t \geq 0$. Then

$$
\bigcap_{t \geq 0} F(T(t))=F(T(1)) \cap F(T(\sqrt{2}))
$$

holds. Using this theorem, we discuss convergence theorems to a common fixed point of $\{T(t): t \geq 0\}$.
\end{abstract}

\section{INTRODUCTION}

Let $C$ be a subset of a Banach space $E$, and let $T$ be a nonexpansive mapping on $C$, i.e., $\|T x-T y\| \leq\|x-y\|$ for all $x, y \in C$. We know that $T$ has a fixed point in the case that $E$ is uniformly convex and $C$ is bounded, closed and convex; see Browder [5], Göhde [9], and Kirk [13. We denote by $F(T)$ the set of all fixed points of $T$.

Let $\tau$ be a Hausdorff topology on $E$. A family of mappings $\{T(t): t \geq 0\}$ is called a one-parameter $\tau$-continuous semigroup of mappings on $C$ if the following are satisfied:

(sg 1) $T(s+t)=T(s) \circ T(t)$ for all $s, t \geq 0$;

(sg 2) for each $x \in C$, the mapping $t \mapsto T(t) x$ from $[0, \infty)$ into $C$ is continuous with respect to $\tau$.

As the topology $\tau$, we usually consider the strong topology of $E$. A family of mappings $\{T(t): t \geq 0\}$ is called a one-parameter $\tau$-continuous semigroup of nonexpansive mappings on $C$ (nonexpansive semigroup, for short) if ( $\mathrm{sg} 1),(\mathrm{sg} 2)$ and the following ( $\mathrm{sg} 3)$ are satisfied:

( $\operatorname{sg} 3)$ for each $t \geq 0, T(t)$ is a nonexpansive mapping on $C$.

We know that $\{T(t): t \geq 0\}$ has a common fixed point in the case that $E$ is uniformly convex and $C$ is bounded, closed and convex; see Browder [5]. Moreover,

Received by the editors December 17, 2003.

2000 Mathematics Subject Classification. Primary 47H20, 47H10.

Key words and phrases. Nonexpansive semigroup, common fixed point, irrational number.

The author was supported in part by Grants-in-Aid for Scientific Research from the Japanese Ministry of Education, Culture, Sports, Science and Technology.

(C)2005 American Mathematical Society Reverts to public domain 28 years from publication 
in 1974, Bruck [8] proved that $\{T(t): t \geq 0\}$ has a common fixed point in the case that $C$ is weakly compact, convex, and has the fixed point property for nonexpansive mappings.

In this paper we prove the following theorem: Let $E$ be a Banach space and let $\tau$ be a Hausdorff topology on $E$. Let $\{T(t): t \geq 0\}$ be a one-parameter $\tau$-continuous semigroup of mappings on a subset $C$ of $E$. Then

$$
\bigcap_{t \geq 0} F(T(t))=F(T(1)) \cap F(T(\sqrt{2}))
$$

holds. Using this theorem, we discuss convergence theorems to a common fixed point of $\{T(t): t \geq 0\}$.

\section{Preliminaries}

Throughout this paper we denote by $\mathbb{Q}$ the set of all rational numbers, and by $\mathbb{N}$ the set of all positive integers. For a real number $t$, we denote by $[t]$ the maximum integer not exceeding $t$. It is obvious that for each real number $t$, there exists $\varepsilon \in[0,1)$ such that $t=[t]+\varepsilon$.

We recall that a Banach space $E$ is called strictly convex if $\|x+y\| / 2<1$ for all $x, y \in E$ with $\|x\|=\|y\|=1$ and $x \neq y$. E is called uniformly convex if for each $\varepsilon>0$, there exists $\delta>0$ such that $\|x+y\| / 2<1-\delta$ for all $x, y \in E$ with $\|x\|=\|y\|=1$ and $\|x-y\| \geq \varepsilon$. It is clear that a uniformly convex Banach space is strictly convex. The norm of $E$ is called Fréchet differentiable if for each $x \in E$ with $\|x\|=1, \lim _{t \rightarrow 0}(\|x+t y\|-\|x\|) / t$ exists and is attained uniformly in $y \in E$ with $\|y\|=1$.

The following lemma is a corollary of Bruck's result in 7 .

Lemma 1 (Bruck [7]). Let $C$ be a subset of a strictly convex Banach space E. Let $S$ and $T$ be nonexpansive mappings from $C$ into $E$ with a common fixed point. Then for each $\lambda \in(0,1)$, a mapping $U$ from $C$ into $E$ defined by $U x=\lambda S x+(1-\lambda) T x$ for $x \in C$ is nonexpansive and $F(U)=F(S) \cap F(T)$ holds.

Proof. It is obvious that $F(U) \supset F(S) \cap F(T)$. Fix $x \in F(U)$ and $w \in F(S) \cap F(T)$. Then we have

$$
\begin{aligned}
\|x-w\| & =\|\lambda S x+(1-\lambda) T x-w\| \\
& \leq \lambda\|S x-w\|+(1-\lambda)\|T x-w\| \\
& \leq \lambda\|x-w\|+(1-\lambda)\|x-w\| \\
& =\|x-w\|
\end{aligned}
$$

and hence

$$
\|x-w\|=\|\lambda S x+(1-\lambda) T x-w\|=\|S x-w\|=\|T x-w\| .
$$

So, from the strict convexity of $E$, we obtain

$$
x=\lambda S x+(1-\lambda) T x=S x=T x .
$$

That is, $x \in F(S) \cap F(T)$. This completes the proof.

The following four convergence theorems for nonexpansive mappings are well known. 


$$
\bigcap_{t \geq 0} F(T(t))=F(T(1)) \cap F(T(\sqrt{2}))
$$

Theorem 1 (Baillon [2]). Let $T$ be a nonexpansive mapping on a bounded closed convex subset $C$ of a Hilbert space $E$. Let $x \in C$ and define a sequence $\left\{x_{n}\right\}$ in $C$ by $x_{n}=\sum_{k=1}^{n} T^{k} x / n$ for $n \in \mathbb{N}$. Then $\left\{x_{n}\right\}$ converges weakly to a fixed point of $T$.

Theorem 2 (Reich [17]). Let $E$ be a uniformly convex Banach space whose norm is Fréchet differentiable. Let $T$ be a nonexpansive mapping on a bounded closed convex subset $C$ of $E$. Define a sequence $\left\{x_{n}\right\}$ in $C$ by $x_{1} \in C$ and $x_{n+1}=$ $\alpha_{n} T x_{n}+\left(1-\alpha_{n}\right) x_{n}$ for $n \in \mathbb{N}$, where $\left\{\alpha_{n}\right\}$ is a sequence in $[0,1]$ satisfying $\sum_{n=1}^{\infty} \alpha_{n}\left(1-\alpha_{n}\right)=\infty$. Then $\left\{x_{n}\right\}$ converges weakly to a fixed point of $T$.

Theorem 3 (Browder [6]). Let $T$ be a nonexpansive mapping on a bounded closed convex subset $C$ of a Hilbert space $E$. Let $\left\{\lambda_{n}\right\}$ be a sequence in $(0,1)$ converging to 0 . Fix $u \in C$ and define a sequence $\left\{x_{n}\right\}$ in $C$ by $x_{n}=\left(1-\lambda_{n}\right) T x_{n}+\lambda_{n} u$ for $n \in \mathbb{N}$. Then $\left\{x_{n}\right\}$ converges strongly to a fixed point of $T$.

Theorem 4 (Wittmann [24]). Let $T$ be a nonexpansive mapping on a bounded closed convex subset $C$ of a Hilbert space $E$. Let $u \in C$ and define a sequence $\left\{x_{n}\right\}$ in $C$ by $x_{1} \in C$ and $x_{n+1}=\left(1-\lambda_{n}\right) T x_{n}+\lambda_{n}$ u for $n \in \mathbb{N}$, where $\left\{\lambda_{n}\right\}$ is a sequence in $[0,1]$ satisfying $\lim _{n} \lambda_{n}=0, \sum_{n=1}^{\infty} \lambda_{n}=\infty$, and $\sum_{n=1}^{\infty}\left|\lambda_{n+1}-\lambda_{n}\right|<\infty$. Then $\left\{x_{n}\right\}$ converges strongly to a fixed point of $T$.

\section{LEMMAS}

In this section we prove two lemmas, which are used in Section 4 .

Lemma 2. Let $t$ be a nonnegative real number and let $\left\{\beta_{n}\right\}$ be a sequence in $(0, \infty)$ converging to 0 . Define sequences $\left\{\delta_{n}\right\}$ in $[0, \infty)$ and $\left\{k_{n}\right\}$ in $\mathbb{N} \cup\{0\}$ as follows:

- $\delta_{1}=t$;

- $k_{n}=\left[\delta_{n} / \beta_{n}\right]$ for $n \in \mathbb{N}$;

- $\delta_{n+1}=\delta_{n}-k_{n} \beta_{n}$ for $n \in \mathbb{N}$.

Then the following hold:

(i) $0 \leq \delta_{n+1}<\beta_{n}$ for all $n \in \mathbb{N}$;

(ii) $k_{n} \in \mathbb{N} \cup\{0\}$ for all $n \in \mathbb{N}$;

(iii) $\left\{\delta_{n}\right\}$ converges to 0 ;

(iv) $\sum_{j=1}^{n} k_{j} \beta_{j}+\delta_{n+1}=t$ for all $n \in \mathbb{N}$;

(v) $\sum_{j=1}^{\infty} k_{j} \beta_{j}=t$.

Proof. We put $\varepsilon_{n} \in[0,1)$ with $\delta_{n} / \beta_{n}=k_{n}+\varepsilon_{n}$ for $n \in \mathbb{N}$. We have

$$
\delta_{n+1}=\delta_{n}-k_{n} \beta_{n}=\varepsilon_{n} \beta_{n}<\beta_{n}
$$

for all $n \in \mathbb{N}$. From this, we also have $\delta_{n+1}=\varepsilon_{n} \beta_{n} \geq 0$. This implies (i). It is obvious that (ii) and (iii) follow from (i). Let us prove (iv) by induction. We have

$$
k_{1} \beta_{1}+\delta_{2}=k_{1} \beta_{1}+\left(\delta_{1}-k_{1} \beta_{1}\right)=\delta_{1}=t .
$$

We assume (iv) holds for some $n \in \mathbb{N}$. Then we have

$$
\begin{aligned}
\sum_{j=1}^{n+1} k_{j} \beta_{j}+\delta_{n+2} & =\sum_{j=1}^{n+1} k_{j} \beta_{j}+\left(\delta_{n+1}-k_{n+1} \beta_{n+1}\right) \\
& =\sum_{j=1}^{n} k_{j} \beta_{j}+\delta_{n+1}=t .
\end{aligned}
$$


So, by induction, we obtain (iv). It is obvious that (v) follows from (iii) and (iv). This completes the proof.

Lemma 3. Let $\alpha$ and $\beta$ be positive real numbers satisfying $\alpha / \beta \notin \mathbb{Q}$. Define sequences $\left\{\alpha_{n}\right\}$ in $(0, \infty)$ and $\left\{k_{n}\right\}$ in $\mathbb{N}$ as follows:

- $\alpha_{1}=\max \{\alpha, \beta\}$;

- $\alpha_{2}=\min \{\alpha, \beta\}$;

- $k_{n}=\left[\alpha_{n} / \alpha_{n+1}\right]$ for all $n \in \mathbb{N}$;

- $\alpha_{n+2}=\alpha_{n}-k_{n} \alpha_{n+1}$ for all $n \in \mathbb{N}$.

Then the following hold:

(i) $0<\alpha_{n+1}<\alpha_{n}$ for all $n \in \mathbb{N}$;

(ii) $k_{n} \in \mathbb{N}$ for all $n \in \mathbb{N}$;

(iii) $\alpha_{n} / \alpha_{n+1} \notin \mathbb{Q}$ for all $n \in \mathbb{N}$;

(iv) $\left\{\alpha_{n}\right\}$ converges to 0 .

Proof. We note that (i) implies (ii). We shall prove (i), (ii) and (iii) by induction. By the assumption of $\alpha / \beta \notin \mathbb{Q}$, we have $\alpha \neq \beta$ and hence

$$
\alpha_{1}=\max \{\alpha, \beta\}>\min \{\alpha, \beta\}=\alpha_{2}>0 .
$$

It is obvious that $\alpha_{1} / \alpha_{2} \notin \mathbb{Q}$. We assume that $0<\alpha_{j+1}<\alpha_{j}$ and $\alpha_{j} / \alpha_{j+1} \notin \mathbb{Q}$ for some $j \in \mathbb{N}$. Since $\alpha_{j+2}=\alpha_{j}-k_{j} \alpha_{j+1}$, we have

$$
\frac{\alpha_{j+2}}{\alpha_{j+1}}=\frac{\alpha_{j}}{\alpha_{j+1}}-k_{j} \notin \mathbb{Q}
$$

and hence $\alpha_{j+1} / \alpha_{j+2} \notin \mathbb{Q}$. Put $\varepsilon_{j} \in[0,1)$ satisfying $\alpha_{j} / \alpha_{j+1}=k_{j}+\varepsilon_{j}$. Since $\alpha_{j} / \alpha_{j+1} \notin \mathbb{Q}$, we note that $\varepsilon_{j}>0$. We have

$$
\alpha_{j+2}=\alpha_{j}-k_{j} \alpha_{j+1}=\varepsilon_{j} \alpha_{j+1}<\alpha_{j+1} .
$$

From this, we also have $\alpha_{j+2}=\varepsilon_{j} \alpha_{j+1}>0$. Therefore we have shown that $0<\alpha_{j+2}<\alpha_{j+1}$ and $\alpha_{j+1} / \alpha_{j+2} \notin \mathbb{Q}$. By induction, we obtain (i), (ii) and (iii). Let us prove (iv). Since $\left\{\alpha_{n}\right\}$ is a sequence of positive real numbers and strictly decreasing, $\left\{\alpha_{n}\right\}$ converges to some $\alpha_{\infty} \in[0, \infty)$. Arguing by contradiction, we assume $\alpha_{\infty}>0$. Then we can choose $j \in \mathbb{N}$ such that

$$
\alpha_{\infty}<\alpha_{j+1}<\alpha_{j}<2 \alpha_{\infty} .
$$

We have

$$
k_{j}=\left[\frac{\alpha_{j}}{\alpha_{j+1}}\right]=1 \quad \text { and } \quad \alpha_{j+2}=\alpha_{j}-k_{j} \alpha_{j+1}=\alpha_{j}-\alpha_{j+1}<\alpha_{\infty} .
$$

This is a contradiction. Therefore $\alpha_{\infty}=0$, that is, we obtain (iv). This completes the proof.

\section{MAin RESUlts}

In this section we give our main results. We first prove the following.

Proposition 1. Let $E$ be a Banach space and let $\tau$ be a Hausdorff topology on $E$. Let $\{T(t): t \geq 0\}$ be a one-parameter $\tau$-continuous semigroup of mappings on a subset $C$ of $E$. Let $\left\{\alpha_{n}\right\}$ be a sequence in $[0, \infty)$ converging to $\alpha_{\infty} \in[0, \infty)$, and satisfying $\alpha_{n} \neq \alpha_{\infty}$ for all $n \in \mathbb{N}$. Suppose that $z \in C$ satisfies $T\left(\alpha_{n}\right) z=z$ for all $n \in \mathbb{N}$. Then $z$ is a common fixed point of $\{T(t): t \geq 0\}$. 


$$
\bigcap_{t \geq 0} F(T(t))=F(T(1)) \cap F(T(\sqrt{2}))
$$

Proof. We note that

$$
T\left(\alpha_{\infty}\right) z=\tau-\lim _{n \rightarrow \infty} T\left(\alpha_{n}\right) z=z .
$$

We put $\beta_{n}=\left|\alpha_{n}-\alpha_{\infty}\right|>0$ for $n \in \mathbb{N}$. By the assumption, $\left\{\beta_{n}\right\}$ is a sequence in $(0, \infty)$ converging to 0 . Since

$$
\max \left\{\alpha_{n}, \alpha_{\infty}\right\}=\min \left\{\alpha_{n}, \alpha_{\infty}\right\}+\beta_{n},
$$

we have

$$
\begin{aligned}
T\left(\beta_{n}\right) z & =T\left(\beta_{n}\right) \circ T\left(\min \left\{\alpha_{n}, \alpha_{\infty}\right\}\right) z \\
& =T\left(\beta_{n}+\min \left\{\alpha_{n}, \alpha_{\infty}\right\}\right) z=T\left(\max \left\{\alpha_{n}, \alpha_{\infty}\right\}\right) z \\
& =z
\end{aligned}
$$

for all $n \in \mathbb{N}$. We also have

$$
T(0) z=T(0) \circ T\left(\alpha_{1}\right) z=T\left(0+\alpha_{1}\right) z=T\left(\alpha_{1}\right) z=z .
$$

Fix $t>0$. Then by Lemma 2, there exists a sequence $\left\{k_{n}\right\}$ in $\mathbb{N} \cup\{0\}$ such that

$$
\sum_{n=1}^{\infty} k_{n} \beta_{n}=t
$$

For each $n \in \mathbb{N}$ with $\sum_{j=1}^{n} k_{j} \beta_{j}>0$, we obtain

$$
\begin{aligned}
T\left(\sum_{j=1}^{n} k_{j} \beta_{j}\right) z & =T\left(\beta_{n}\right)^{k_{n}} \circ T\left(\beta_{n-1}\right)^{k_{n-1}} \circ \cdots \circ T\left(\beta_{2}\right)^{k_{2}} \circ T\left(\beta_{1}\right)^{k_{1}} z \\
& =T\left(\beta_{n}\right)^{k_{n}} \circ T\left(\beta_{n-1}\right)^{k_{n-1}} \circ \cdots \circ T\left(\beta_{2}\right)^{k_{2}} z \\
& =\cdots=T\left(\beta_{n}\right)^{k_{n}} z \\
& =z
\end{aligned}
$$

where $T\left(\beta_{j}\right)^{0}$ is the identity mapping on $C$. This implies

$$
T(t) z=\tau \text { - } \lim _{n \rightarrow \infty} T\left(\sum_{j=1}^{n} k_{j} \beta_{j}\right) z=z .
$$

This completes the proof.

Now, we prove one of our main results.

Proposition 2. Let $E$ be a Banach space and let $\tau$ be a Hausdorff topology on E. Let $\{T(t): t \geq 0\}$ be a one-parameter $\tau$-continuous semigroup of mappings on a subset $C$ of $E$. Let $\alpha$ and $\beta$ be positive real numbers satisfying $\alpha / \beta \notin \mathbb{Q}$. Then

$$
\bigcap_{t \geq 0} F(T(t))=F(T(\alpha)) \cap F(T(\beta))
$$

holds.

Proof. It is obvious that

$$
\bigcap_{t \geq 0} F(T(t)) \subset F(T(\alpha)) \cap F(T(\beta)) .
$$


So we shall prove the converse inclusion. We fix $z \in F(T(\alpha)) \cap F(T(\beta))$. Define sequences $\left\{\alpha_{n}\right\}$ in $(0, \infty)$ and $\left\{k_{n}\right\}$ in $\mathbb{N}$ as in Lemma 3. By the assumption, we have

$$
T\left(\alpha_{1}\right) z=T(\max \{\alpha, \beta\}) z=z \quad \text { and } \quad T\left(\alpha_{2}\right) z=T(\min \{\alpha, \beta\}) z=z .
$$

If $T\left(\alpha_{j}\right) z=T\left(\alpha_{j+1}\right) z=z$, then we have

$$
T\left(\alpha_{j+2}\right) z=T\left(\alpha_{j+2}\right) \circ T\left(\alpha_{j+1}\right)^{k_{j}} z=T\left(\alpha_{j+2}+k_{j} \alpha_{j+1}\right) z=T\left(\alpha_{j}\right) z=z .
$$

So, by induction, we obtain $T\left(\alpha_{n}\right) z=z$ for all $n \in \mathbb{N}$. Since $\left\{\alpha_{n}\right\}$ is a sequence of positive real numbers converging to 0 , Proposition 1 yields that $z$ is a common fixed point of $\{T(t): t \geq 0\}$. This completes the proof.

As a direct consequence of Proposition 2, we obtain the following.

Corollary 1. Let E, $\tau, C$ and $\{T(t): t \geq 0\}$ be the same as in Proposition 2 . Then

$$
\bigcap_{t \geq 0} F(T(t))=F(T(1)) \cap F(T(\sqrt{2}))
$$

holds.

Using Lemma 1, we obtain the following.

Corollary 2. Let E, $\tau, C, \alpha$ and $\beta$ be the same as in Proposition 2 , Let $\{T(t)$ : $t \geq 0\}$ be a $\tau$-continuous semigroup of nonexpansive mappings on $C$. Assume that $E$ is strictly convex, and $F(T(\alpha)) \cap F(T(\beta)) \neq \varnothing$. Then

$$
\bigcap_{t \geq 0} F(T(t))=\{z \in C: \lambda T(\alpha) z+(1-\lambda) T(\beta) z=z\}
$$

holds for every $\lambda \in(0,1)$.

Corollary 3. Let $E, \tau, C, \alpha$ and $\beta$ be the same as in Proposition 2 , Let $\{T(t)$ : $t \geq 0\}$ be a $\tau$-continuous semigroup of nonexpansive mappings on $C$. Assume that $E$ is uniformly convex, and $C$ is bounded, closed and convex. Then

$$
\bigcap_{t \geq 0} F(T(t))=\{z \in C: \lambda T(\alpha) z+(1-\lambda) T(\beta) z=z\}
$$

holds for every $\lambda \in(0,1)$.

\section{Convergence theorems}

Several authors have studied convergence theorems for nonexpansive semigroups; see [1, 3, 11, 16, 18, 20, 22] and others. For example, Suzuki and Takahashi in 22. proved the following: Let $C$ be a compact convex subset of a Banach space $E$ and let $\{T(t): t \geq 0\}$ be a strongly continuous semigroup of nonexpansive mappings on $C$. Define a sequence $\left\{x_{n}\right\}$ in $C$ by $x_{1} \in C$ and

$$
x_{n+1}=\frac{\lambda}{t_{n}} \int_{0}^{t_{n}} T(s) x_{n} d s+(1-\lambda) x_{n}
$$

for $n \in \mathbb{N}$, where $\lambda$ is a constant in $(0,1)$, and $\left\{t_{n}\right\}$ is a sequence in $(0, \infty)$ satisfying $\lim _{n} t_{n}=\infty$ and $\lim _{n} t_{n+1} / t_{n}=1$. Then $\left\{x_{n}\right\}$ converges strongly to a common fixed point of $\{T(t): t \geq 0\}$. 


$$
\bigcap_{t \geq 0} F(T(t))=F(T(1)) \cap F(T(\sqrt{2}))
$$

Using Proposition 2 we can prove many convergence theorems for nonexpansive semigroups. In this section, we state some of them. In the following theorems, we make the assumption:

- Let $E$ be a Banach space and let $\tau$ be a Hausdorff topology on $E$. Let $\{T(t): t \geq 0\}$ be a one-parameter $\tau$-continuous semigroup of nonexpansive mappings on a bounded closed convex subset $C$ of $E$. Let $\alpha$ and $\beta$ be positive real numbers satisfying $\alpha / \beta \notin \mathbb{Q}$.

We first state the following, which are connected with Baillon's type iteration [2]; see pages 63 and 83 in [23].

Theorem 5. Assume that $E$ is a Hilbert space. Let $x \in C$ and define a sequence $\left\{x_{n}\right\}$ in $C$ by

$$
x_{n}=\frac{\sum_{k=1}^{n} \sum_{\ell=1}^{n} T(k \alpha+\ell \beta) x}{n^{2}}
$$

for $n \in \mathbb{N}$. Then $\left\{x_{n}\right\}$ converges weakly to a common fixed point of $\{T(t): t \geq 0\}$.

Proof. We note that

$$
\sum_{k=1}^{n} \sum_{\ell=1}^{n} T(k \alpha+\ell \beta) x=\sum_{k=1}^{n} \sum_{\ell=1}^{n} T(\alpha)^{k} \circ T(\beta)^{\ell} x .
$$

Thus, $\left\{x_{n}\right\}$ converges weakly to a common fixed point $z$ of $T(\alpha)$ and $T(\beta)$. Such $z$ is a common fixed point of $\{T(t): t \geq 0\}$ by Proposition 2 This completes the proof.

Theorem 6. Assume that $E$ is a Hilbert space. Let $x \in C$ and define a sequence $\left\{x_{n}\right\}$ in $C$ by

$$
x_{n}=\frac{\sum_{k=1}^{n}\left(\frac{T(\alpha)+T(\beta)}{2}\right)^{k} x}{n}
$$

for $n \in \mathbb{N}$. Then $\left\{x_{n}\right\}$ converges weakly to a common fixed point of $\{T(t): t \geq 0\}$.

Proof. By Theorem 1] $\left\{x_{n}\right\}$ converges weakly to some $z \in C$ which is a fixed point of $(T(\alpha)+T(\beta)) / 2$. By Corollary 3. we obtain that $z$ is a common fixed point of $\{T(t): t \geq 0\}$. This completes the proof.

We next state the following, which are connected with Krasnosel'skiu-Mann's type iteration [14, 15]; see Reich [17] and Suzuki [19, 21].

Theorem 7. Assume that $E$ is a uniformly convex Banach space whose norm is Fréchet differentiable. Fix $\kappa, \lambda>0$ with $\kappa+\lambda<1$. Define a sequence $\left\{x_{n}\right\}$ in $C$ by $x_{1} \in C$ and

$$
x_{n+1}=\kappa T(\alpha) x_{n}+\lambda T(\beta) x_{n}+(1-\kappa-\lambda) x_{n},
$$

for $n \in \mathbb{N}$. Then $\left\{x_{n}\right\}$ converges weakly to a common fixed point of $\{T(t): t \geq 0\}$. Proof. By Theorem 2] $\left\{x_{n}\right\}$ converges weakly to some $z \in C$ which is a fixed point of

$$
\frac{\kappa}{\kappa+\lambda} T(\alpha)+\frac{\lambda}{\kappa+\lambda} T(\beta) .
$$

So, by Corollary 3, we obtain that $z$ is a common fixed point of $\{T(t): t \geq 0\}$. This completes the proof. 
Theorem 8. Assume that $C$ is compact. Fix $\lambda \in(0,1)$. Define a sequence $\left\{x_{n}\right\}$ in $C$ by $x_{1} \in C$ and

$$
x_{n+1}=\lambda \frac{\sum_{k=1}^{n} \sum_{\ell=1}^{n} T(k \alpha+\ell \beta) x_{n}}{n^{2}}+(1-\lambda) x_{n},
$$

for $n \in \mathbb{N}$. Then $\left\{x_{n}\right\}$ converges strongly to a common fixed point of $\{T(t): t \geq 0\}$.

Using Ishikawa's result in [12, we obtain the following.

Theorem 9. Assume that $C$ is compact. Fix $\kappa, \lambda \in(0,1)$. Define a sequence $\left\{x_{n}\right\}$ in $C$ by $x_{1} \in C$ and

$$
x_{n+1}=(\lambda T(\alpha)+(1-\lambda) I) \circ(\kappa T(\beta)+(1-\kappa) I)^{n} x_{n}
$$

for $n \in \mathbb{N}$, where $I$ is the identity mapping on $C$. Then $\left\{x_{n}\right\}$ converges strongly to a common fixed point of $\{T(t): t \geq 0\}$.

We next state the following, which is connected with Browder's type implicit iteration [6]. We note that $x \mapsto(1-\lambda) T x+\lambda u$ is a contractive mapping if $T$ is a nonexpansive mapping and $\lambda \in(0,1)$. Therefore, the Banach contraction principle 4 yields that such a mapping has a unique fixed point.

Theorem 10. Assume that $E$ is a Hilbert space. Fix $u \in C$ and define a sequence $\left\{x_{n}\right\}$ in $C$ by

$$
x_{n}=\frac{1-\lambda_{n}}{2} T(\alpha) x_{n}+\frac{1-\lambda_{n}}{2} T(\beta) x_{n}+\lambda_{n} u
$$

for $n \in \mathbb{N}$, where $\left\{\lambda_{n}\right\}$ is a sequence in $(0,1)$ converging to 0 . Then $\left\{x_{n}\right\}$ converges strongly to a common fixed point of $\{T(t): t \geq 0\}$.

We finally state the following, which is connected with Halpern's type explicit iteration [10]; see Wittmann [24].

Theorem 11. Assume that $E$ is a Hilbert space. Fix $u \in C$ and define a sequence $\left\{x_{n}\right\}$ in $C$ by $x_{1} \in C$ and

$$
x_{n+1}=\frac{1-\lambda_{n}}{2} T(\alpha) x_{n}+\frac{1-\lambda_{n}}{2} T(\beta) x_{n}+\lambda_{n} u
$$

for $n \in \mathbb{N}$, where $\left\{\lambda_{n}\right\}$ is a sequence in $[0,1]$ satisfying the following:

$$
\lim _{n \rightarrow \infty} \lambda_{n}=0 ; \quad \sum_{n=1}^{\infty} \lambda_{n}=\infty ; \quad \text { and } \quad \sum_{n=1}^{\infty}\left|\lambda_{n+1}-\lambda_{n}\right|<\infty .
$$

Then $\left\{x_{n}\right\}$ converges strongly to a common fixed point of $\{T(t): t \geq 0\}$.

\section{REFERENCES}

[1] S. Atsushiba and W. Takahashi, "Strong convergence theorems for one-parameter nonexpansive semigroups with compact domains", in Fixed Point Theory and Applications, Volume 3 (Y. J. Cho, J. K. Kim and S. M. Kang Eds.), pp. 15-31, Nova Science Publishers, New York, 2002. MR2081406 (2005i:47081)

[2] J. B. Baillon, "Un théorème de type ergodique pour les contractions non linéaires dans un espace de Hilbert", C. R. Acad. Sci. Paris, Sér. A-B, 280 (1975), 1511-1514. MR0375009 (51:11205)

[3] J. B. Baillon, "Quelques properiétés de convergence asymptotique pour les semigroupes de contractions impaires", C. R. Acad. Sci. Paris, 283 (1976), A75-A78. MR0425702 (54:13655) 


$$
\bigcap_{t \geq 0} F(T(t))=F(T(1)) \cap F(T(\sqrt{2}))
$$

[4] S. Banach, "Sur les opérations dans les ensembles abstraits et leur application aux équations intégrales", Fund. Math., 3 (1922), 133-181.

[5] F. E. Browder, "Nonexpansive nonlinear operators in a Banach space", Proc. Nat. Acad. Sci. USA, 54 (1965), 1041-1044. MR0187120 (32:4574)

[6] F. E. Browder, "Convergence of approximants to fixed points of nonexpansive nonlinear mappings in Banach spaces", Arch. Ration. Mech. Anal., 24 (1967), 82-90. MR0206765 $(34: 6582)$

[7] R. E. Bruck, "Properties of fixed-point sets of nonexpansive mappings in Banach spaces", Trans. Amer. Math. Soc., 179 (1973), 251-262. MR0324491 (48:2843)

[8] R. E. Bruck, "A common fixed point theorem for a commuting family of nonexpansive mappings", Pacific J. Math., 53 (1974), 59-71. MR0361945 (50:14387)

[9] D. Göhde, "Zum Prinzip def kontraktiven Abbildung”, Math. Nachr., 30 (1965), 251-258. MR0190718 (32:8129)

[10] B. Halpern, "Fixed points of nonexpanding maps", Bull. Amer. Math. Soc., 73 (1967), 957961. MR 0218938 (36:2022)

[11] N. Hirano, "Nonlinear ergodic theorems and weak convergence theorems", J. Math. Soc. Japan, 34 (1982), 35-46. MR0639803 (83d:47059)

[12] S. Ishikawa, "Common fixed points and iteration of commuting nonexpansive mappings", Pacific J. Math., 80 (1979), 493-501. MR0539430(80g:47065)

[13] W. A. Kirk, "A fixed point theorem for mappings which do not increase distances", Amer. Math. Monthly, 72 (1965), 1004-1006. MR0189009 (32:6436)

[14] M. A. Krasnosel'skiı̌, "Two remarks on the method of successive approximations" (in Russian), Uspehi Mat. Nauk 10 (1955), 123-127. MR0068119 (16:833a)

[15] W. R. Mann, "Mean value methods in iteration", Proc. Amer. Math. Soc., 4 (1953), 506-510. MR0054846 (14:988f)

[16] I. Miyadera and K. Kobayasi, "On the asymptotic behaviour of almost-orbits of nonlinear contraction semigroups in Banach spaces", Nonlinear Anal., 6 (1982), 349-365. MR0654811 (84c:47064)

[17] S. Reich, "Weak convergence theorems for nonexpansive mappings in Banach spaces", J. Math. Anal. Appl., 67 (1979), 274-276. MR0528688(80d:47090)

[18] N. Shioji and W. Takahashi, "Strong convergence theorems for asymptotically nonexpansive semigroups in Hilbert spaces", Nonlinear Anal., 34 (1998), 87-99. MR1631657(99e:47071)

[19] T. Suzuki, "Strong convergence theorem to common fixed points of two nonexpansive mappings in general Banach spaces", J. Nonlinear Convex Anal., 3 (2002), 381-391. MR.1947106 (2003i:47066)

[20] T. Suzuki, "On strong convergence to common fixed points of nonexpansive semigroups in Hilbert spaces", Proc. Amer. Math. Soc., 131 (2003), 2133-2136. MR1963759 (2004b:47108)

[21] T. Suzuki, "Common fixed points of two nonexpansive mappings in Banach spaces", Bull. Austral. Math. Soc., 69 (2004), 1-18. MR2040045 (2004m:47146)

[22] T. Suzuki and W. Takahashi, "Strong convergence of Mann's type sequences for oneparameter nonexpansive semigroups in general Banach spaces", J. Nonlinear Convex Anal., 5 (2004), 209-216. MR2083912 (2005c:47093)

[23] W. Takahashi, "Nonlinear Functional Analysis", Yokohama Publishers, Yokohama, 2000. MR:1864294(2002k:47001)

[24] R. Wittmann, "Approximation of fixed points of nonexpansive mappings", Arch. Math. (Basel), 58 (1992), 486-491. MR1156581 (93c:47069)

Department of Mathematics, Kyushu Institute of Technology, Sensuicho, Tobata, KITAKYUSHU 804-8550, JAPAN

E-mail address: suzuki-t@mns.kyutech.ac.jp 